\title{
TECNOLOGIA UBER NA CIDADE DE MANAUS: \\ MOBILIDADE URBANA, REDES E CIBERCIDADES
}

\begin{tabular}{c} 
UBER TECHNOLOGY IN MANAUS CITY: \\
URBAN MOBILITY, NET AND CYBERCITIES \\
\hline TECNOLOGÍA UBER EN LA CIUDAD DE MANAUS: \\
MOVILIDAD URBANA, REDES Y CIBERCIDADES
\end{tabular}

Soraya Nurieh Auzier Costa ${ }^{1}$

\section{RESUMO}

O presente texto é uma resenha da dissertação de Mestrado defendida no Programa de Pós-Graduação em Ciência da Comunicação da Universidade Federal do Amazonas, intitulada Estudo ecossistêmico sobre o aplicativo uber na cidade de Manaus: mobilidade urbana, redes e cibercidades, de Soraya Nurieh Auzier Costa, sob orientação do professor Dr. Jonas da Silva Gomes Júnior. O estudo, de abordagem ecossistêmica, busca compreender a partir da visão ecossistêmica as interferências causadas pela chegada do aplicativo Uber na cidade de Manaus em abril de 2017 e como esse serviço tecnológico de mobilidade urbana tem proporcionado novas dinâmicas na sociedade manauara, tendo por bases a perspectiva dos Ecossistemas Comunicacionais e apoio da Teoria Ator-Rede. O estudo observa como essas tecnologias de comunicação estão configurando e reconfigurando os espaços urbanos, as relações e práticas sociais, possibilitando analisar os processos de virtualização das cidades a partir da presença dessas tecnologias digitais via aplicações na mediação de prestações de serviços e nos levando a questionar a manutenção das políticas públicas na manutenção dos demais serviços de transportes públicos que devem servir a sociedade no geral.

PALAVRAS-CHAVE: Redes. Tecnologia. Ecossistemas comunicacionais. Cibercidades.

\section{ABSTRACT}

This text is a review of the Master's thesis defended in the Post-Graduate Program in Communication Science at the Federal University of Amazonas, entitled Ecosystem study on the uber application in the city of Manaus: urban mobility, nets and cybercities, by Soraya Nurieh Auzier Costa, under the guidance of Professor Dr. Jonas da Silva Gomes Júnior. The study, with an ecosystem approach, seeks to understand from the ecosystem view the interference caused by the arrival of the Uber application in the city of Manaus in April 2017 and how this technological urban mobility service has provided new dynamics in the society of Manaus, based on perspective of Communicational Ecosystems and support from Actor-Network Theory. The study observes how these communication technologies are configuring and reconfiguring urban spaces, relationships and social practices, making it possible to analyze the virtualization processes of cities based on the presence of these digital technologies via applications in the mediation of service provision and leading us to question the maintenance of public policies in the maintenance of other public transport services that should serve society in general.

KEYWORDS: Nets. Technology. Communicational ecosystems. Cybercities.

Submetido em: 10/11/2020- Aceito em: 07/12/2020 - Publicado em: 25/01/2021

${ }^{1}$ Mestre pelo Programa de Pós-Graduação em Ciências da Comunicação da Universidade Federal do Amazonas (PPGCCOM/UFAM). E-mail: sorayacomunicacao@gmail.com Lattes:

http://lattes.cnpq.br/8488295033950563 


\section{RESUMEN}

Este texto es una revisión de la tesis de maestría defendida en el Programa de Posgrado en Ciencias de la Comunicación de la Universidad Federal de Amazonas, titulada Estudio ecosistémico sobre la aplicación uber en la ciudad de Manaus: movilidad urbana, redes y ciberciudades, por Soraya Nurieh Auzier Costa, bajo la guía del profesor Dr. Jonas da Silva Gomes Júnior. El estudio, con enfoque ecosistémico, busca comprender desde la perspectiva ecosistémica la interferencia provocada por la llegada de la aplicación Uber a la ciudad de Manaus en abril de 2017 y cómo este servicio tecnológico de movilidad urbana ha proporcionado nuevas dinámicas en la sociedad de Manaus, a partir de perspectiva de los ecosistemas comunicacionales y apoyo de la teoría actor-red. El estudio observa cómo estas tecnologías de la comunicación están configurando y reconfigurando los espacios urbanos, las relaciones y las prácticas sociales, permitiendo analizar los procesos de virtualización de las ciudades a partir de la presencia de estas tecnologías digitales a través de aplicaciones en la mediación de la prestación de servicios y llevándonos a cuestionar el mantenimiento de políticas públicas en el mantenimiento de otros servicios de transporte público que deben servir a la sociedad en general.

PALABRAS CLAVE: Redes. Tecnología. Ecosistemas comunicacionales. Ciberciudades.

\section{INTRODUZINDO A PESQUISA}

A dissertação de Mestrado defendida no Programa de Pós-Graduação em Ciências da Comunicação da Universidade Federal do Amazonas, intitulada Estudo ecossistêmico sobre o aplicativo uber na cidade de Manaus: mobilidade urbana, redes e cibercidades, de Soraya Nurieh Auzier Costa, sob orientação do professor Dr. Jonas da Silva Gomes Júnior. O estudo objetivou compreender como o serviço tecnológico Uber voltado à mobilidade urbana possibilitou novas dinâmicas sociais na capital Amazonense com sua chegada em abril de 2017, tendo por base a perspectiva dos Ecossistemas Comunicacionais e apoio da Teoria Ator-Rede.

A visão ecossistêmica abre a possibilidade de desvendar os ecossistemas camuflados, pois a cidade se mostra muitas vezes como uma única materialidade sistematiza, encobrindo muitas vezes fenômenos e características próprias, que podem contribuir em maiores estratégias, métodos e ponderações sobre o contexto como um todo. Ao adotar a perspectiva dos ecossistemas comunicacionais sob essa estrutura, auxilia na visualização dos processos comunicacionais e possíveis mudanças de um cenário de paradigmas entre o encontro de novas tecnologias em sociedade e as conexões existentes dentro das redes estabelecidas dentro do espaço da cidade.

Os ecossistemas comunicacionais abarcam dentro de si uma visão ecológica dos sistemas em redes, comunicacionais e das complexidades existentes dentro das sociedades, as quais estão todos interligados de forma dinâmica. Em âmbito nacional a perspectiva ecossistêmica tem sido desenvolvida no Programa de Pós-Graduação em Ciências da Comunicação da Universidade Federal do Amazonas (UFAM) em sua área de concentração para os estudos da comunicação, sob a perspectiva da complexidade sistêmica e 
informacional dos fenômenos comunicativos.

Cardoso, Nogueira e Martins (2017) expõem que a abordagem ecossistêmica comunicacional está para além do entendimento clássico da comunicação como um fenômeno centrado na emissão, transmissão e recepção de informação. A visão ecossistêmica abre possibilidades, pois desvenda ecossistemas camuflados, suas interferências, trocas e dessa forma possibilita realizar ponderações, estratégias e métodos sobre as interconexões.

A dissertação é dividida em quatro partes. Na primeira é apresentada uma revisão de literatura com análises de diversas pesquisas em diferentes áreas acadêmicas sobre o que já foi publicado sobre a empresa de tecnologia Uber. Posteriormente, uma breve explanação sobre aplicativos de mobilidade, a história da startup Uber e seus processos legislativos. Na segunda parte, tem como objetivo discorrer sobre tecnologia em sociedade e apresentar os conceitos teóricos que compõem a presente pesquisa como: o conceito de Redes, Ecossistemas Comunicacionais, Cibercidades e a Teoria Ator-Rede. Na terceira, é definido o que se entende por mobilidade urbana, acessibilidade, distinguindo os conceitos e suas aplicações. Na última contém a estrutura metodológica, métodos de investigação utilizados na pesquisa, definido o corpus para a realização da análise e resultados da pesquisa, as categorias de análise que surgiram a partir da análise de conteúdo. Nesse processo foi possível visualizar os sistemas estabelecidos pela tecnologia Uber na sociedade Manauara e construir ilustrações a partir da visão ecossistêmica e da Teoria Ator-Rede, seguido das considerações finais.

\section{O PROCESSO DE VIRTUALIZAÇÃO DO ESPAÇO URBANO}

Fruto de um trabalho coletivo, a cidade é construção da sociedade, no processo da materialização da história de um povo, suas relações sociais, suas dinâmicas políticas, econômicas e religiosas. Esta construção surge a partir da necessidade humana em pertencer, se agregar, se organizar em torno do bem estar comum, criando seus laços, onde possa se manter e estabelecer as suas conexões.

Suas estruturas existentes devem ser voltadas para a população, tornando o espaço urbano um lugar de encontro, trocas, circulação, igualdades e para usufruto do patrimônio, sendo um local onde seja garantido o direito constitucional à cidade e à cultura. Com a possibilidade de conhecer, desfrutar da cultura e das estruturas disponíveis, através de um deslocamento fácil, seguro para todos os usuários, com a possibilidade de permanência para que essa fruição aconteça.

Sendo de obrigação dos dirigentes públicos buscarem um sistema de mobilidade mais igualitário do ponto de vista social, com sustentação financeira e ao mesmo tempo sem excluir os mais pobres, além de gerar o mínimo de externalidades negativas possíveis. 
(CARVALHO, 2016)

As cidades por serem sistemas complexos e em redes, nas suas formas sociais e na criação de infra-estrutura, onde nascem, crescem e se desenvolvem a partir de diversos fatores sociais, culturais, políticos e tecnológicos. Esses sistemas complexos estão passando por profundas modificações com a entrada de dispositivos de comunicação e tecnologias de informação em seu contexto urbano, de forma que possibilite o processo das cibercidades, conhecidas também como cidades digitais.

Com o desenvolvimento de diversos dispositivos digitais, a expansão das redes, a intensificação e cotidianização dos usos de novas tecnologias de informação e de comunicação que as primeiras cibercidades surgiram como a expressão digital da era da informação (Pires, 2003).

As cibercidades são as cidades da era da globalização, onde os aparatos digitais, redes telemáticas, a transmissão de dados e serviços tecnológicos fazem parte da vida quotidiana do espaço urbano, se integrando como a infra-estrutura existente, com as novas tecnologias de comunicação e informação as quais imprimem novas marcas ao urbano com serviços, acesso e trazendo à sociedade novas forma de distribuição do fluxo informacional.

Surgem para atender as demandas da comunidade presentes nas redes digitais, virtualmente criadas de acordo com as necessidades de uma comunidade real e crescem a partir de algumas iniciativas que visam caracterizar estes espaços virtuais no ambiente offline, não se limitando apenas como um meio de implementação de serviços a serem fornecidos e acessados, pois a cidade independente da estrutura tecnologia permanece sendo um espaço da sociabilidade, de manifestação do poder e lutas de pertencimento.

A informatização da sociedade vem transformando as práticas sociais, os modos de vivência, os formatos de produção e o consumo das informações. Desenvolvendo-se de forma onipresente, onde os usuários não precisam mais se deslocarem até a rede, mas a rede em uma conexão generalizada é quem passa a envolver os usuários e os objetos (LEMOS, 2005), devido a grande densidade de tecnologias digitais móveis presentes nas cidades contemporâneas como celulares smartphones, laptops, palms e as redes de acesso à internet, criando assim novas dinâmicas sociologias de deslocamento e transitar, modificando as estruturas urbanas, emergindo as cidades da mobilidade.

As cibercidades devem ser vistas como um processo de virtualização das cidades (Lemos, 2001), formas espaço-temporais que se constroem pelo movimento e união de dois elementos: transporte e comunicação. Demonstrando que a era da conexão e a era da mobilidade caminham lado a lado, onde a comunicação móvel com suas flexibilidades está redefinindo as condutas e fluxos dos lugares tradicionais como ruas, praças, avenidas, demais localidades das cidades, transformando esses lugares em lugares digitais.

O processo tecnológico e de virtualização em sociedade necessitam serem pensada de maneira sistêmica, da mesma forma que novas espécies introduzidas dentro de um ecossistema, a transferência descomedida de tecnologias pode resultar no aparecimento de 
mais impactos negativos para a sociedade do que desejáveis, podendo vir a desestabilizá-la (VERASZTO et al, 2009), pois algumas dinâmicas em rede e tecnologias, as quais muitas vezes são criadas com objetivos e intuitos de empreendedores reais, em suas iniciativas que visam adentrar nos espaços das relações econômicas e culturais das cidades capitalistas em conjunto da dimensão digital. As formações das novas cidades informacionais, resultam também do crescimento de novas relações econômicas e de produção, formadas pela consolidação de novas redes de trabalho entre os espaços.

Essas estruturas nos territórios não se tratam somente de influências digitais ou popularização de aparatos tecnológicos, mas sim dos constantes processos da própria sociedade, das suas construções, com a interferência dos eventos globais, do consumo, prestações de serviços que buscam auxiliar e oferecer meios através de tecnologias, trazendo consigo apontamentos de alguns problemas e potencialidades para os espaços urbanos.

Esse processo norteará as tomadas de decisões de empresas, governos e cidadãos, com o intuito de tornar as atividades urbanas mais eficientes e sustentáveis nas esferas econômica, social, ecológica e política. Consequentemente, o foco hoje são projetos que visam tornar a economia, a mobilidade urbana, o meio ambiente, os cidadãos e o governo mais inteligentes.

A tecnologia está inserida de tal forma que molda os estilos de vida das pessoas, modificando todo um conjunto de costumes e valores, agrega-se ao dia a dia dos espaços urbanos, nas relações e na cultura de um povo, contribuindo para o entendimento dos processos de criação da própria humanidade e dos contextos sociais, sendo a tecnologia também fonte de conhecimentos e com novos saberes, concebida em função de novas demandas e exigências da própria sociedade, em suas dinâmicas de transmutação contínua, de forma cada vez mais veloz, principalmente pela interferência e utilização das novas tecnologias, de forma que não é mais possível tratá-las como um fenômeno isolado da dinâmica social.

Muitos são os desafios ainda a serem enfrentados para a implementação, planejamento e desenvolvimento desses espaços urbanos como exclusão digital, os elevados custos dos serviços tradicionais de telefonia, barreira ao acesso à internet, a ausência de provedores de internet nas pequenas localidades, oferta concentrada nos grandes centros urbanos, os preços dos equipamentos de informática em relação as condições socioeconômicas dominantes, falta de capacitação profissional para lidar com as novas tecnologias de informação e comunicação.

No contexto das cibercidades, a Uber participa como um ator no processo de virtualização do cenário urbano, onde as novas tecnologias de informações adentram oferecendo serviços mediados por aplicações digitais, com dinâmicas alinhadas ao contexto virtual e infraestruturas tecnológicas, reconfigurando o cotidiano das cidades a partir do protagonismo dos softwares nas mais diversas atividades humanas, iniciando uma era construída a partir de códigos, dando forma a um contexto urbano onde o digital se faz presente nas mais diversas práticas urbanas. 
A instauração dessas novas dinâmicas instituídas pela emergência das novas tecnologias de comunicação e das redes telemáticas reconfigurando os espaços urbanos em cibercidades, segundo Lemos (2004), as não devem ser interpretadas como a criação de uma nova cidade em detrimento das existentes e sim essas novas dinâmicas, dispositivos e redes telemáticas cada vez mais presentes nas práticas sociais das cidades.

Entretanto, o processo de virtualização e a densidade tecnológica que o contexto urbano apresenta nas cibercidades devem ser encarados com seriedade, defende Lemos (2013), pois podem apresentar contradições e efeitos colaterais.

\section{RESULTADOS}

A abordagem metodológica adotada, seus aspectos, métodos e procedimentos, como as questões da pesquisa foram tratadas e como se buscou responder ao problema da investigação que visou estudar as interconexões criadas em sociedade a partir da chegada do aplicativo de mobilidade urbana Uber na capital amazonense.

Em busca de descrever os principais desdobramentos e interferências em redes, foram analisadas 30 matérias do ano de 2017, através de coleta de informação em três portais jornalísticos de notícia da cidade de Manaus: Em Tempo, D24AM e Jornal A Crítica. A amostragem foi feita de forma aleatória, levando em conta o total de 92 matérias publicadas nesse período nos portais escolhidos. As categorias surgidas a partir da análise de conteúdo se configuram como as impressões obtidas acerca da realidade estudada e os efeitos da chegada do serviço tecnológico. O que possibilitou a obtenção de respostas para a pesquisa e visualização das interconexões estabelecidas pelo aplicativo ao ser inserido no contexto ecossistêmico da cidade de Manaus, não somente na estrutura urbana, mas também no contexto econômico, tecnológico, cultural e social.

A visão ecossistêmica permitiu desenvolver a ilustração abaixo: 


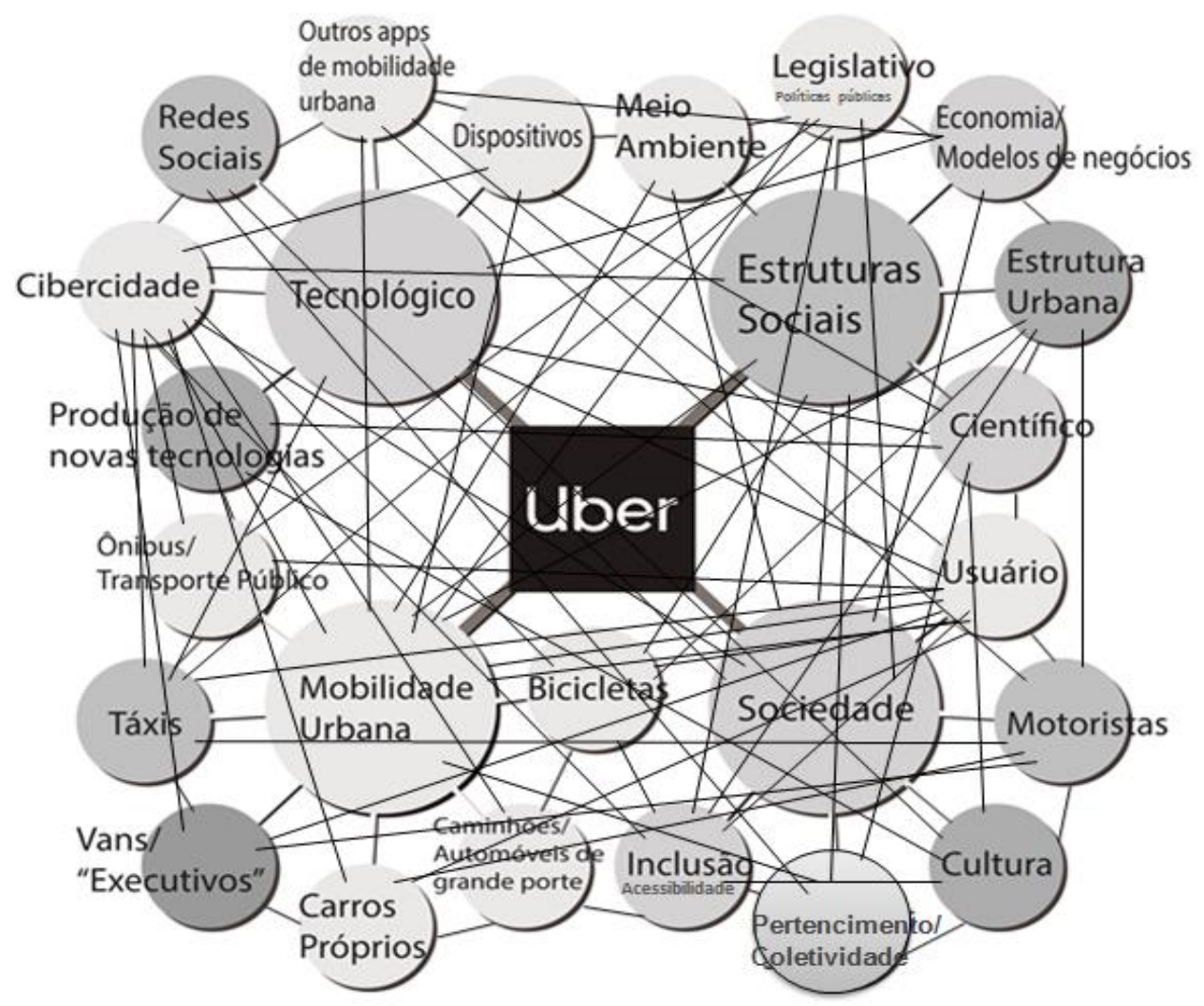

Figura 1: Rede Ecossistêmica.

Fonte: Elaborado pela autora, com base na pesquisa (2019).

\section{Tecnológico}

O cenário da tecnologia se refere às estruturas tecnológicas presentes na cidade, desde estruturas urbanas que comportam a tecnologia virtual sendo apliadas nas ruas para assumirem novas funções e coma presença dos serviços tecnológicos de mobilidade gerando densidade e fluxos virtuais dentro do espaço urbano, condições essa da criação das cibercidades.

Quando a Uber adentrou na cidade outros aplicativos concorrentes surgiram juntos e até mesmo criações de aplicativos locais de aplicativos de mobilidade, incentivando o progresso da criação, programação e tecnológica da cidade. As influências até mesmo de forma abstrata e subjetiva da ideia de tecnologia, como fonte de incentivo para $o$ aparecimento de novos dispositivos ou aparatos, adentrando como fatores de efeitos da chegada dessa nova tecnologia na cidade. 
Dispositivos movéis: tornando-se um mecanismo de mobilidade urbana.

Outros apps de mobilidade urbana: surgindo, chegando a cidade, concorrências e criações locais.

Redes sociais digitais, mídias digitais e grupos no whatsapp propagadores de informações e fatos sobre o aplicativo.

Cibercidade: possibilitando e incentivando a construção da estrutura tecnológica social.

Produção de novas tecnologias: incentivo a produção tecnológica local.

Quadro 1: Categorização dos cenários que a tecnologia Uber adentra na cidade.

Fonte: Elaborado pela autora, com base na pesquisa (2019).

\section{Mobilidade Urbana}

Os diversos atores que surgiram no cenário da mobilidade urbana foram os modais de transporte presentes nas ruas, não somente os de transporte público, os de carga também adentraram. Formando o ecossistema do ambiente físico que o aplicativo adentra.

\begin{tabular}{|l|}
\multicolumn{1}{|c|}{ Cenário da Mobilidade Urbana } \\
\hline Onibus/Transporte Público \\
\hline Táxis \\
\hline Vans/Executivos \\
\hline Carros próprios \\
\hline Caminhões/Automóveis de grande porte \\
\hline Bicicletas \\
\hline Motos \\
\hline Mototáxis \\
\hline
\end{tabular}

Quadro 2: Categorização dos cenários que a tecnologia Uber adentra na cidade.

Fonte: Elaborado pela autora, com base na pesquisa (2019).

\section{Estruturas Sociais}

O sistema em rede denominado estruturas sociais estão ligadas as questões de gestão pública, como cenários presentes em sociedade que influenciam a subsistência como a economia, o meio ambiente, estruturas urbanas e o legislativo.

\section{Estruturas Sociais}

Meio Ambiente: menos carros nas ruas, menos poluentes para as cidades. Quantidade de carros nas ruas, influência.

Legislativo: necessidade de uma regulamentação, interferindo em leis.

Economia/Modelos de Negócios: novo serviço, emprego, menos gastos para o usuário com o transporte.

Estrutura Urbana: compartilhamento de carros possibilitando a presença de menos veículos nas ruas.

Científico: produções científicas voltadas as problemática em volta do aplicativo.

Segurança pública.

Quadro 3: Categorização dos cenários que a tecnologia Uber adentra na cidade.

Fonte: Elaborado pela autora, com base na pesquisa (2019) 


\section{Sociedade}

O sistema referente à sociedade se refere à população, as pessoas em geral e nas relações de pertencimento, desde o imaginário até as práticas que propagam entre si e nos demais sistemas.

\begin{tabular}{|l|}
\multicolumn{1}{c|}{ Sociedade } \\
\hline Usuário: agente de manutenção/principal ator para implementação da tecnologia uber. \\
\hline Motoristas: agente de manutenção/principal ator para implementação da tecnologia uber. \\
\hline Cultura: mudanças de hábitos e relação com o transporte. \\
\hline Pertencimento: Coletividade \\
\hline Inclusão: possibilidade de classes a utilização de um transporte que julga de qualidade e mais em conta. \\
\hline
\end{tabular}

Quadro 4: Categorização dos cenários que a tecnologia Uber adentra na cidade.

Fonte: Elaborado pela autora, com base na pesquisa (2019).

A compreensão dos sistemas e de redes é importante para a formação do conceito dos ecossistemas comunicacionais, onde a rede se interliga a comunicação e englobando os sistemas, tornando o fenômeno comunicacional complexo e dessa forma a comunicação se apresenta de uma perspectiva participativa, com a interação de todos os envolvidos, possibilitando um processo que reconhece a importância de cada indivíduo do grupo.

Essa visão possibilidade de desvendar os ecossistemas camuflados, pois a cidade se mostra muitas vezes como uma única materialidade sistematiza, encobrindo muitas vezes fenômenos e características próprias, que podem contribuir em maiores estratégias, métodos e ponderações sobre o contexto como um todo. Cenários e estruturas da sociedade que se apresentam como elementos fundamentais para compreensão dos desdobramentos comunicacionais do aplicativo na sociedade Manauara. Esses sistemas envolvidos compõe o ecossistema em torno da tecnologia Uber na cidade.

\section{CONSIDERAÇÕES FINAIS}

O presente estudo se propôs a observar a cidade como uma grande rede que estava recebendo uma tecnologia com um formato que antes não existia, a mobilidade urbana via aplicação digital e perceber as novas dinâmicas com a interferência desse novo ator.

Um dos principais resultados que a dissertação obteve foi ao observar o espaço urbano, os sistemas de transportes e os diversos núcleos sociais, nos deparamos com divergências, que nos leva a percepção de avanços tecnológicos que servem a marcas e empresas e do outro problemas sociais, como os serviços básicos em sociedade que ficaram parados no tempo, em completo abandono e debilidades, fazendo com que a sociedade em geral não tenha o mesmo progresso que a tecnologia digital, a qual deveriam caminhar alinhados. 
Em alguns casos os negócios e a inovação tecnológica nos projetos das cidades inteligentes pode levar à privatização do espaço público, à mecanização do cidadão em função da estrutura burocrática, ou à adoção de soluções automatizadas para o espaço urbano que atendam mais aos interesses de empresas do que da população (Lemos, 2013).

Trazendo a reflexão do tipo de sociedade que nós queremos viver e para onde estamos caminhando, fazendo necessário um alinhamento entre essa densidade tecnológica e as necessidades da sociedade em geral.

A contribuição da dissertação é a percepção de que a tecnologia estudada na pesquisa se trata de um produto, a qual faz parte de um mercado, que visa seus lucros como qualquer outro produto, desmistificando o que já foi apresentado no mercado da inovação como "soluções tecnológicas", pois se trata de algo que não necessariamente criam uma cidade mais acessível apenas por oferecer preços mais baratos em uma conexão em redes digitais, pois é algo muito ausente em outras realidades socioeconômicas dentro do mesmo contexto urbano, por se tratar de um serviço mercadológico de uma empresa privada e não uma política pública.

O desenvolvimento de políticas públicas e de infra-estruturas tecnológicas são passos estratégicos que proporcionaram avanços a sociedade, com a participação efetiva dos cidadãos no manuseio de informações, conhecimentos, o barateamento dos custos e o acesso a serviços digitais. Isto significa o estabelecimento de relações que promovam a democratização e a inserção de múltiplos segmentos sociais e culturais na rede, possibilitando não só uma sociabilidade de seus membros, mas a ampliação dos tradicionais processos capitalistas de produção, circulação e consumo.

Para a sociedade, a mobilidade urbana vem sendo construída através de políticas de desenvolvimento urbano, articulações, união de políticas de transporte, circulação, acessibilidade e trânsito. Ficando cada vez mais evidente que ações de valorização do espaço público têm reflexo direto no desenvolvimento urbano. Somente o aumentar de investimento financeiro visando à criação de infraestruturas não garante a melhoria da mobilidade urbana, pois as infraestruturas devem estar inter-relacionadas com um planejamento sistêmico, o qual deve abranger outros setores da sociedade que serão influenciados direta e indiretamente, para que dessa forma se produzam benefícios efetivos e proporcionais aos recursos empregados.

\section{REFERÊNCIAS}

CARDOSO, Leigiane Alves; NOGUEIRA, Wilson Souza; MARTINS, Rosemeire. Ecossistemas Comunicacionais e as Relações com a Interdisciplinaridade. Revista Eletrônica Mutações, [S.1.], v. 8, n. 14, p. 314-320, jun. 2017. ISSN 2178-7018. Disponível em: <http://www.periodicos.ufam.edu.br/relem/article/view/3602>. Acesso em: 18 mar. 2018. 
CARVALHO, Carlos Henrique Ribeiro. Desafios da mobilidade urbana no Brasil, Texto para Discussão, No. 2198, Instituto de Pesquisa Econômica Aplicada (IPEA), Brasília. 2016.

LEMOS, André. Cibercidades. In: LEMOS, André; PALACIOS, Marcos (Org.) Janelas do ciberespaço: comunicação e cibercultura. Porto Alegre: Sulina, 2001.

LEMOS,Andre, Cibercultura, tecnologia e vida social na cultura contemporânea. Porto Alegre, Sulina, 2004.

LEMOS, André. Cibercultura e mobilidade: a era da conexão.In: XXVIII Congresso Brasileiro De Ciências Da Comunicação, 2005, Rio de Janeiro. Disponível em: <http://www.intercom.org.br/papers/nacionais/2005/resumos/r1465-1.pdf>. Acesso em: 19 de set. de 2018.

LEMOS, André. Cidades inteligentes. GVexecutivo, v. 12 , nº 2, jul/dez. 2013. Disponível em: 〈http://bibliotecadigital.fgv.br/ojs/index.php/gvexecutivo/article/view/20720/19454>. Acesso em: 14 ago. 2019.

PIRES, Hindenburgo Francisco. Inovação Tecnológica e Desenvolvimento da Cibercidade: $O$ advento da Cibercidade. Simpósio Internacional Cybercity, São Paulo, 2003. Disponível em: <http://www.cibergeo.org/artigos/CYBERCITY2003.pdf>. Acesso em: 14 ago. 2019.

VERASZTO, E. V.; SILVA, D.; MIRANDA, N. A.; SIMON, F. O. Tecnologia: buscando uma definição para o conceito. Prisma.com (Portugual), n. 8, p. 19-46, 2009. Disponível em: <https://pentaho.letras.up.pt/ojs/index.php/prismacom/article/view/2065/1901>. Acesso em: 20 ago. 2019.

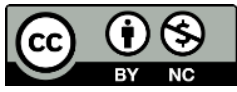

Este é um artigo de acesso aberto distribuído sob os termos da Licença Creative Commons Atribuição Não Comercial-Compartilha Igual (CC BYNC- 4.0), que permite uso, distribuição e reprodução para fins não comerciais, com a citação dos autores e da fonte original e sob a mesma licença. 